\title{
VETERINARY EPIDEMIOLOGY
}

\section{Vaccination strategies for foot-and-mouth disease}

\section{Arising from: M. J. Tildesley et al. Nature 440, 83-86 (2006)}

When foot-and-mouth disease struck the United Kingdom in 2001, the traditional 'stamping out' policy of 1967-68 was supplemented by the pre-emptive culling of animals in premises contiguous to infected premises. A model proposed by Tildesley et al. ${ }^{1}$ indicates that the introduction of vaccination should at least halve the number of premises that would need to be subjected to culling in the event of another outbreak. We contest, however, that the overlapping confidence intervals of the outputs of their model, and the inconsistency of their results compared with those from previous models, call into question the model's value as a decision tool, while adding little to the recognized tenet of ring vaccination.

Foot-and-mouth disease occurred regularly in the United Kingdom over the first twothirds of the twentieth century ${ }^{2}$. As a result, a 'stamping out' policy was developed that was based on movement restrictions, enforcement of biosecurity, rapid slaughter of animals from infected premises, and tracing and slaughter of dangerous contacts. A disease-free period of more than 30 years followed the epidemic of 1967-68. When the disease returned in 2001, this 'stamping out' policy was augmented by pre-emptive culling in premises contiguous to infected premises; vaccination was not used.

Tildesley et al. ${ }^{1}$ model 'reactive' vaccination as an alternative to pre-emptive culling on contiguous premises and find that this strategy would at least halve the number of premises for culling. They explore different strategies and conclude that the impact of an epidemic can be restricted most effectively by using reactive ring vaccination, prioritizing farms closest to infected premises. The authors claim that this technique would be more effective than the prioritization of high-risk farms. They also conclude that the addition of culling in contiguous premises is not advantageous when vaccination is practised. However, the overlapping confidence intervals of the parameters associated with the impact of the epidemic under different strategies (Table 2 of ref. 1) indicate that the model cannot offer a clear choice between different scenarios, making its value as a decision tool questionable.

The value of this modelling approach in decision support is further undermined because the conclusions of previous papers based on the same model ${ }^{3,4}$ are inconsistent with those of Tildesley et al. ${ }^{1}$. For example, Keeling et al. ${ }^{3}$ showed that ring vaccination of cattle would reduce the total cull by only about $15 \%$, and that removing culling in contiguous premises from a control strategy that includes ring vaccination would have doubled the total cull; Keeling et al. ${ }^{4}$ also pointed out that, although ring vaccination generates some benefit, even $10-\mathrm{km}$ rings reduce the size of the epidemic by only about $20 \%$, and that "vaccinating the nearest farms [to infected premises] would be futile", suggesting that reactive vaccination should be targeted to farms that are predicted to contribute most to future spatial transmission of disease.

The model used by the authors, here ${ }^{1}$ and previously ${ }^{3,4}$, is parameterized in such a way as to favour disease-control methods (culling, vaccination or both) that are pre-emptive and localized ${ }^{5}$. Farms are assumed to become instantly and maximally infectious four days before the simulated infection is reported. The spatial simulation of disease spread in the models is based on spread-tracing information, which the authors themselves previously recognized ${ }^{3}$ as likely to be biased towards overestimating the amount of short-distance spread of disease. The model still needs to be validated against data not used in its construction $^{6}$. Without this validation, it remains an untested hypothesis that has produced inconsistent and imprecise conclusions about vaccination policy, and therefore adds little, if anything, to the already well established principle of ring vaccination ${ }^{7}$.

Richard P. Kitching ${ }^{\star}$, Nicholas M. Taylor', Michael V. Thrusfield $;$

*National Centre for Foreign Animal Diseases, Winnipeg R3E 3M4, Canada

†Veterinary Epidemiology and Economics

Research Unit, University of Reading, Earley Gate, Reading RG6 6AR, UK

Veterinary Clinical Sciences, University of

Edinburgh, Easter Bush Veterinary Centre, Roslin

EH25 9RG, UK

m.thrusfield@ed.ac.uk

1. Tildesley, M. J. et al. Nature 440, 83-86 (2006).

2. HMSO Report of the Committee of Enquiry on Foot-andMouth Disease 1968 Vols 1, 2 (Her Majesty's Stationery Office, London, 1969).

3. Keeling, M. J. et al. Science 294, 813-817 (2001).

4. Keeling, M. J., Woolhouse, M. E. J., May, R. M., Davies, G. \& Grenfell, B. T. Nature 421, 136-142 (2003)

5. Kitching, R. P., Thrusfield, M. V. \& Taylor, N. M. Rev. Sci. Tech. 25, 293-311 (2006).

6. Spedding, C. R. W. An Introduction to Agricultural Systems 2nd edn (Elsevier Applied Science, London, 1988).

7. Donaldson, A. I. \& Doel, T. R. Vet. Rec. 131, 114-120 (1992).

Competing financial interests: declared none. doi: 10.1038 /nature05604

\section{VETERINARY EPIDEMIOLOGY}

\section{Tildesley et al. reply}

Replying to: R. P. Kitching, N. M. Taylor \& M. V. Thrusfield Nature 445, doi: 10.1038/nature05604 (2007)

Kitching et al. ${ }^{1}$ express concerns about our mathematical models of reactive vaccination strategies for the control of foot-and-mouth disease epidemics ${ }^{2}$. However, in our view, these concerns are misplaced.

Kitching et al. note that simulated epidemic sizes can be highly variable, but they misconstrue the reason for this. The variability does not imply a lack of precision in the outputs of the model; rather, the model is capturing a well recognized feature of outbreaks of infectious diseases in general $^{3}$ and of foot-and-mouth disease in particular ${ }^{4}$. By performing tens of thousands of simulations, we can robustly establish the relative effectiveness of different control options on average across the whole range of expected outcomes. We regard this as a major strength of our approach: it would be most unwise to recommend control policies that were potentially vulnerable to expected stochastic variability. Our approach also allows us to consider alternative criteria for an effec- tive control policy, such as minimizing the probability of a very large epidemic.

These results are not inconsistent with our earlier work ${ }^{5,6}$ (Kitching et al. are quoting this out of context), in which we considered different scenarios - specifically, different start dates for vaccination. Previous results can be recovered readily by using the latest version of the model. The novelty of our recent study ${ }^{2}$ lies in the finding that, in a resource-limited situation, considerable and unanticipated gains in 